\title{
Competing endogenous RNA analysis reveals the regulatory potency of circRNA_036186 in HNSCC
}

\author{
WEN-LONG WANG ${ }^{1,2}$, ZHI YANG $^{1,2}$, YI-JUAN ZHANG ${ }^{3}$, PING LU $^{1,2}$, \\ YOU-KANG NI ${ }^{1,2}$, CHANG-FU SUN ${ }^{1,2}$ and FA-YU LIU ${ }^{1,2}$ \\ Departments of ${ }^{1}$ Oromaxillofacial-Head and Neck Surgery, and ${ }^{2}$ Oral and Maxillofacial Surgery, \\ School of Stomatology, China Medical University, Shenyang, Liaoning 110002; ${ }^{3}$ Department of Anesthesiology, \\ The First Affiliated Hospital, China Medical University, Shenyang, Liaoning 110002, P.R. China
}

Received January 26, 2018; Accepted June 26, 2018

DOI: 10.3892/ijo.2018.4499

\begin{abstract}
This study aimed to characterize circular RNA (circRNA) expression profiles and biological functions in head and neck squamous cell carcinoma (HNSCC). Differentially expressed circRNAs were screened using an Arraystar Human CircRNA Array and verified by reverse transcription-quantitative polymerase chain reaction. Multiple bioinformatics methods and a hypergeometric test were employed to predict the interactions between RNAs and the functional circRNA-microRNA (miRNA)-mRNA axes in HNSCC. As a result, 287 circRNAs and 1,053 mRNAs were determined to be differentially expressed in HNSCC compared with the adjacent tissue. In addition, the expression levels of circRNA_036186 and tyrosine 3-monooxygenase/tryptophan 5-monooxygenase
\end{abstract}

Correspondence to: Dr Fa-Yu Liu or Dr Chang-Fu Sun, Department of Oromaxillofacial-Head and Neck Surgery, School of Stomatology, China Medical University, 117 Nanjing Bei Jie, Heping, Shenyang, Liaoning 110002, P.R. China

E-mail:1fyhjk@126.com

E-mail: changfusun@hotmail.com

Abbreviations: circRNA, circular RNA; miRNA or miR, microRNA; ncRNA, non-coding RNA; RT-qPCR, reverse transcription-quantitative polymerase chain reaction; GO, Gene Ontology; KEGG, Kyoto Encyclopedia of Genes and Genomes; MRE, miRNA response element; ceRNA, competing endogenous RNA; UTR, untranslated region; RBP, RNA-binding protein; TCGA, The Cancer Genome Atlas; BLCA, bladder urothelial carcinoma; BRCA, breast invasive carcinoma; HNSCC, head and neck squamous cell carcinoma; LUAD, lung adenocarcinoma; LUSC, lung squamous cell carcinoma; UCEC, uterine corpus endometrial carcinoma; YAP, yes-associated protein 1; TAZ, WW domain-containing transcription regulator protein 1; 14-3-3\}, tyrosine 3-monooxygenase/tryptophan 5-monooxygenase activation protein, $\zeta$ polypeptide; HDAC6, histone deacetylase 6; SPOCK1, sparc/osteonectin, cwcv and kazal-like domains proteoglycan 1; CDK6, cyclin-dependent kinase 6

Key words: circular RNA, microRNA, competing endogenous RNA, microarray, head and neck squamous cell carcinoma

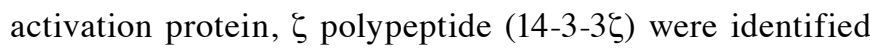
to be significantly different. A competing endogenous RNA (ceRNA) network was constructed, consisting of 5 circRNAs, 385 miRNAs and 96 mRNAs. Furthermore, we predicted that miR-193b-3p exerts a significant effect on 14-3-3 and was significantly associated with the Hippo signaling pathway in HNSCC. On the whole, these findings suggest that circRNA_036186 likely regulates 14-3-3 $\zeta$ expression by functioning as a ceRNA in the development and progression of HNSCC.

\section{Introduction}

Head and neck squamous cell carcinoma (HNSCC) is the sixth most common type of cancer worldwide, with $\sim 600,000$ new cases diagnosed each year. Treatment protocols include surgery, chemotherapy and radiation, all of which may result in major physical and psychological effects on the patient (1). Despite continuing research and advances in treatment, the clinical outcomes and overall survival rates of patients with HNSCC have not improved significantly over the past several decades, with an overall 5-year survival rate of $60 \%(2,3)$. A high rate of metastasis is a characteristic of HNSCC, which highlights the urgent need for the identification of novel biomarkers to more accurately predict recurrence and metastasis in patients with HNSCC.

MicroRNAs (miRNAs or miRs) are endogenous short non-coding RNAs (ncRNAs) that bind to the complementary seed sequence on the 3'-untranslated region (UTR) of specific target protein-coding genes, known as miRNA response elements (MREs), thereby resulting in the inhibition of target gene expression (4). Increasing evidence suggests that miRNA dysregulation is associated with the tumorigenesis and progression of various cancer types (5-8). It has been demonstrated that certain protein-coding genes and their pseudogene sequences contain the same conserved MREs in their 3'UTRs, and their respective expression levels may be regulated through competing for the same miRNAs through these MREs (9). A hypothesis regarding these RNA transcripts, termed competing endogenous RNAs (ceRNAs), has been proposed (10). According to this hypothesis, MREs can be seen as an 'RNA language' through which transcripts can 
communicate with each other to regulate their respective expression levels. Any RNA transcript with MREs may function as a ceRNA and compete with other RNA transcripts with similar MREs for the same miRNAs.

Circular RNAs (circRNAs) are widespread and diverse endogenous ncRNAs with covalently closed circular structures $(11,12)$. There are three main types of circRNAs based on their biogenesis patterns, including exonic circRNAs (13), intronic circRNAs $(14,15)$ and exon-intron circRNAs $(15-17)$, which are transcribed from pre-mRNA sequences by RNA polymerase (Pol) II (18). circRNAs are predominantly generated by reverse splicing between the specific and conservative sequences in upstream and downstream regions, respectively, a process known as back-splicing $(19,20)$. Of the three types, exonic circRNAs account for the largest proportion, and are abundant in the cytoplasm (21-23). There are multiple conserved MREs on circRNAs, known as miRNA sponges, that sequester miRNAs from binding to targets on mRNAs, thereby enhancing the expression of certain genes $(24,25)$. However, studies have demonstrated that only a few circRNAs have multiple MREs for specific miRNAs, most of which contain only one or two MREs $(26,27)$. Intronic circRNAs are mainly present in the nucleus and have almost no enrichment in MREs. They largely accumulate around the transcription initiation sites of their parental genes through interaction with elongation RNA Pol II machinery, serving a cis-regulatory role on their parental coding genes (14). Exonintron circRNAs are abundant in the nucleus, and interact with small nuclear ribonucleoprotein (U1 snRNP) and Pol II at the promoter regions of genes to promote the transcription of their parental genes (16). At present, studies on circRNAs are mainly focused on exonic circRNAs. Their interactions with disease-related miRNAs indicate that circular RNAs are important factors in disease regulation $(25,28)$. For instance, the circRNA ciRS-7 contains multiple and conservative miRNA-7 binding sites, thus acting as an efficient miRNA sponge to adsorb miRNA-7, and thus inhibiting its function (29).

Increasing evidence has demonstrated that ciRS-7 plays a crucial role in the tumorigenesis and progression of cancers through its effect on the level of miRNA-7, thus mediating gene expression via ceRNA mechanisms (30). Given the widespread involvement of miR-7 in various cancer-related pathways and its potential regulatory functions in Parkinson's and Alzheimer's disease, ciRS-7 may play a key role in other neurological disorders and tumorigenesis (24). Exploring the mechanisms underlying the interactions between these ceRNAs may add a new dimension to our understanding of the mechanisms of cancer, and may potentially provide a novel approach to cancer treatment.

The molecular mechanisms of circRNAs in the carcinogenesis and progression of HNSCC are largely uncharacterized thus far. Herein, we hypothesized that differentially expressed circRNAs could participate in the tumorigenesis and metastasis of HNSCC. To explore the roles of circRNAs, we examined the different expression patterns of circRNAs and mRNAs in HNSCC using microarray analyses. Subsequently, based on our microarray data and the circRNA-miRNA-mRNA interactions predicted with Arraystar's miRNA target prediction software based on TargetScan (31) and miRanda (32), a network was constructed using Cytoscape $(33,34)$. OncomiR (35) and UALCAN (36) are online resources from The Cancer Genome Atlas (TCGA) specifically for miRNA and protein-coding gene analysis across many cancer types, respectively. DIANA-miRPath v3.0 (37) is an efficient tool to analyze the combinatorial effect of miRNAs on target pathways by P-value. According to the results obtained using these three tools, we predicted that circRNA_036186 may promote the expression of tyrosine 3-monooxygenase/tryptophan

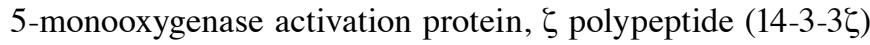
by functioning as a sponge for the inhibitory miR-193b-3p. Our data indicate that circRNA_036186 may be suitable as a novel diagnostic marker and therapeutic target in HNSCC, as well as providing a basis for further studies regarding the role of circRNAs in HNSCC.

\section{Materials and methods}

Patients and samples. This study was approved and supervised by The Ethics Committee of China Medical University (Shenyang, China). Five pairs of HNSCC and para-carcinoma tissues were collected from patients that had not received pre-operative chemotherapy or radiotherapy who underwent resective surgery at the Head and Neck Tumor Center (School of Stomatology, China Medical University). Written informed consent was obtained from all patients. All samples were obtained during surgery and immediately stored in liquid nitrogen. The diagnosis of HNSCC was confirmed by a pathological examination (Table I).

RNA extraction. Total RNA was extracted from the HNSCC and para-carcinoma tissue samples using TRIzol reagent (Invitrogen/Thermo Fisher Scientific, Inc., Waltham, MA, USA), according to the manufacturer's instructions. Subsequently, the RNA quantity and quality was measured using an ND-1000 system (NanoDrop; Thermo Fisher Scientific, Inc., Wilmington, DE, USA). RNA integrity was assessed via standard denaturing agarose gel electrophoresis methods.

Microarray assay. Five pairs of carcinoma and para-carcinoma tissues were used for a microarray assay, with the intention of identifying differentially expressed circRNAs and mRNAs by comparing the HNSCC samples with the adjacent control samples. Sample preparation and microarray hybridization were performed based on Arraystar's standard protocols. Briefly, total RNAs were digested with RNase R (Epicentre; Illumina, Inc., San Diego, CA, USA) to remove linear RNAs and enrich for circRNAs. Subsequently, the enriched circRNAs were amplified and transcribed into fluorescent cRNA, utilizing a random priming method (Arraystar Super RNA Labeling kit; Arraystar Inc., Rockville, MD, USA). The labeled cRNAs were hybridized onto the Arraystar Human circRNA Array V2 (8x15K; Arraystar Inc.). After washing the slides, the arrays were scanned using an Agilent G2505C scanner (Agilent Technologies, Inc., Santa Clara, CA, USA). Agilent Feature Extraction software (version 11.0.1.1) was used to analyze the acquired array images. The raw data were quantile normalized, and subsequent data processing was performed using the limma package in R. Significantly differentially 
Table I. Clinicopathological characteristics of the five patients.

\begin{tabular}{|c|c|c|c|c|c|c|}
\hline $\begin{array}{l}\text { Patient } \\
\text { no. }\end{array}$ & Sex & Age & Position & $\begin{array}{l}\text { Pathological } \\
\text { diagnosis }\end{array}$ & $\begin{array}{l}\text { Histological } \\
\text { grade }\end{array}$ & $\begin{array}{l}\text { TNM } \\
\text { stage }\end{array}$ \\
\hline 1 & Male & 53 & $\begin{array}{l}\text { Abdomen of the tongue and } \\
\text { bottom of the oral cavity }\end{array}$ & HNSCC & G2 & T2N0M0 \\
\hline 3 & Male & 33 & Abdomen of the tongue & HNSCC & G2 & T2N0M0 \\
\hline 6 & Female & 63 & Lateral border of tongue & HNSCC & $\mathrm{G} 2$ & $\mathrm{~T} 2 \mathrm{~N} 1 \mathrm{M} 0$ \\
\hline 8 & Male & 59 & Lateral border of tongue & HNSCC & $\mathrm{G} 2$ & $\mathrm{~T} 2 \mathrm{~N} 1 \mathrm{M} 0$ \\
\hline 9 & Male & 43 & Lateral border of tongue & HNSCC & $\mathrm{G} 1-\mathrm{G} 2$ & $\mathrm{~T} 2 \mathrm{~N} 1 \mathrm{M} 0$ \\
\hline
\end{tabular}

HNSCC, head and neck squamous cell carcinoma.

expressed circRNAs and mRNAs between the two groups were identified through volcano plot filtering with the thresholds of $\mathrm{P}<0.05$ and the Benjamini-Hochberg false discovery rate (FDR) of $<0.5$. Differentially expressed circRNAs and mRNAs between the HNSCC and adjacent control groups were identified through fold change filtering (circRNAs, llog fold changel $>1.5$; mRNAs, llog fold changel $>2$ ). Hierarchical clustering was performed to show the characteristics of the expression profiles based on the values of all expressed transcripts, and differentially expressed transcripts.

Gene Ontology (GO) and pathway analyses. The GO project provides a controlled vocabulary to describe gene and gene product attributes in any organism (http://www.geneontology. org/). The GO categories cover three different domains: Biological process (BP), cellular component (CC) and molecular function (MF). The P-value denotes the significance of GO terms enrichment in the differentially expressed genes. The Kyoto Encyclopedia of Genes and Genomes (KEGG) pathway analysis was used for harvesting pathway clusters covering the molecular interaction and reaction networks in differential gene expression profile. The P-value denotes the significance of the pathway association with the specified genes.

Competing endogenous RNA network analysis. The potential miRNA response elements were searched on the sequences of circRNAs and mRNAs, besides a measure with the number of common miRNAs, a hypergeometric test was executed for each ceRNA pair separately (38).

The overlap of the same miRNA seed sequence binding site both on the circRNA and mRNA was predicted with the following parameters: miRNA coverage $\geq 0.1$; context $+<$ -4.999999977648258e-2; species = human; common Num $\geq 1$; ceRNA type $=$ protein coding; structure $>140 ; \mathrm{P}<0.05$; context <-4.999999977648258e-2; energy <-10. miRNA coverage describes the threshold value for both SeqMiRNA coverage and CeMiRNA coverage. Context+ is the threshold value of the sum of the context+ scores used in TargetScan from version 6.0. Common Num is the threshold value of the number of common miRNAs between the ceRNA gene and the gene of interest. Structure is the threshold value of the sum of the structure scores used in miRanda. P-value is the threshold for the P-values calculated using hypergeometric tests. Context is the threshold value of the sum of the context scores used in TargetScan before version 5.x. Energy is the threshold value of the sum of the free energy predicted by miRanda.

Exploring miRNA dysregulation in tumor development and progression. miRNA dysregulation in tumor development and progression was predicted using OncomiR (www.oncomir.org (35) based on TCGA across 30 cancer types. Significance in tumor development was determined through a paired Student's t-test between normal and tumor tissues; and for survival, an unpaired Student's t-test and univariate Cox proportional hazards analysis between living and deceased patients.

Prediction of potentially functional circRNA-miRNA-mRNA axes. The miRNA pathway investigation was carried out based on DIANA-miRPath v3.0 (http://www.microrna.gr/ miRPathv3) (37). Firstly, we searched miRNA candidates in DIANA-miRPath v3.0-DIANA-TarBase v7.0 (39) and microT-CDS v5.0 $(40,41)$ to predict the potential target genes and pathways with thresholds of $\mathrm{P}<0.05$ and MicroT score $>0.8$. Subsequently, elaborated signaling pathway networks based on KEGG database were constructed gathering target genes and interacting proteins. Finally, the expression differences for target genes in malignant tumors were analyzed using UALCAN (http://ualcan.path.uab.edu) (36).

Reverse transcription-quantitative polymerase chain reaction $(R T-q P C R)$ validation. The microarray results were confirmed by RT-qPCR with the same RNA samples as those used for microarray analysis. Following RNA isolation, SuperScript ${ }^{\mathrm{TM}}$ III Reverse Transcriptase (Invitrogen/Thermo Fisher Scientific, Inc., Waltham, MA, USA) was used to synthesize the cDNA according to the manufacturer's instructions. Subsequently, qPCR was performed using a ViiA 7 Real-time PCR System (Applied Biosystems/Thermo Fisher Scientific, Inc., Foster City, CA, USA) with a total reaction volume of $10 \mu$, including $0.5 \mu \mathrm{l}$ PCR forward primer $(10 \mu \mathrm{M}), 0.5 \mu \mathrm{l}$ PCR reverse primer (10 $\mu \mathrm{M}), 2 \mu \mathrm{l}$ cDNA, $5 \mu \mathrm{l} 2 \mathrm{X}$ Master Mix and $2 \mu \mathrm{l}$ double distilled water. The thermocycling conditions were as follows: $95^{\circ} \mathrm{C}$ for $10 \mathrm{~min}$, then $10 \mathrm{sec}$ at $95^{\circ} \mathrm{C}$ and $60 \mathrm{sec}$ at $60^{\circ} \mathrm{C}$ for a total of 40 cycles. $\beta$-actin was used as an internal reference. The experiments were run using three independent wells per 

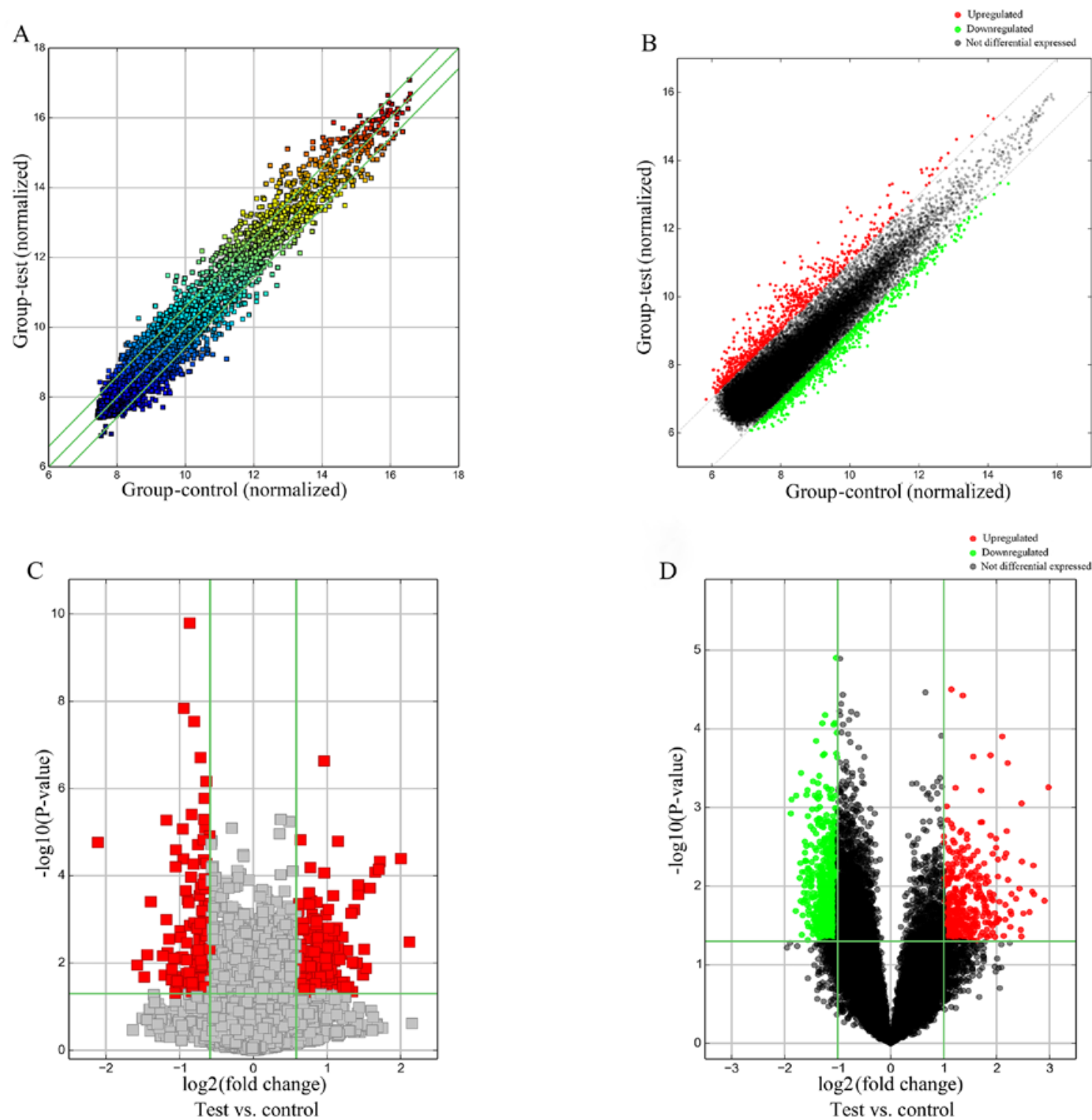

Figure 1. Differences in circRNA and mRNA expression profiles between HNSCC tissues and tumor-adjacent tissues. (A) Scatter plot of circRNAs: The values on the $\mathrm{x}$ - and $\mathrm{y}$-axes are the averaged normalized signal values of groups of samples (log2-scaled). The green lines are fold change lines. The circRNAs above the top line and below the bottom line indicate $>1.5$-fold change between the two compared samples. (B) Scatter plot of mRNAs: The fold change cut-off was 2.0. (C) Volcano plot of circRNAs: The vertical lines correspond to 1.5 -fold up and down, respectively, and the horizontal line represents the P-value cut-off of 0.05 . The red points in the plot represent the differentially expressed circRNAs with statistical significance. (D) Volcano plot of mRNAs: The red and green points represent the differentially expressed mRNAs based on the thresholds of fold change $>2.0$ and $\mathrm{P}<0.05$. circRNA, circular RNA; HNSCC, head and neck squamous cell carcinoma.

condition. For quantitative analyses, the relative expression levels of circRNAs and mRNAs were calculated using the $2^{-\Delta \Delta C q}(42)$ method. The primers used were as follows: circRNA_036186 forward, 5'-CTGAAGCACCGCCCA GCT-3' and reverse, 5'-GACGAGCCACATTCATTCCAG-3'; 14-3-3 forward, 5'-TGTTGTAGGAGCCCGTAG-3' and reverse, 5'-GCAACCTCAGCCAAGTAA-3'; $\beta$-actin $(\mathrm{H})$ forward, 5'-GTGGCCGAGGACTTTGATTG-3' and reverse, 5'-CCT GTAACAACGCATCTCATATT-3'.

Statistical analysis. All data are expressed as the means \pm SD. The statistical significance of the differentially expressed genes as determined by RT-qPCR was estimated using the Student's t-test. $\mathrm{P}<0.05$ was considered to indicate a statistically significant difference.

\section{Results}

Identification of differentially expressed circRNA and $m R N A$ profiles by microarray assay. High-throughput microarray analysis was performed to detect circRNA and mRNA expression in the HNSCC tissues. In the present study, a total of 12,366 circRNAs and 35,252 mRNAs were detected by microarray probes in five paired HNSCC and normal tissues. As a result, 287 dysregulated circRNAs were detected. Among these, 146 and 141 circRNAs were upregulated and downregulated, respectively. Additionally, 1,053 mRNAs were found to be differentially regulated, among which 377 mRNAs were upregulated, while 676 mRNAs were downregulated. Scatter and Volcano Plots, tools for visualizing differential expression between two compared groups, were produced; the former was 


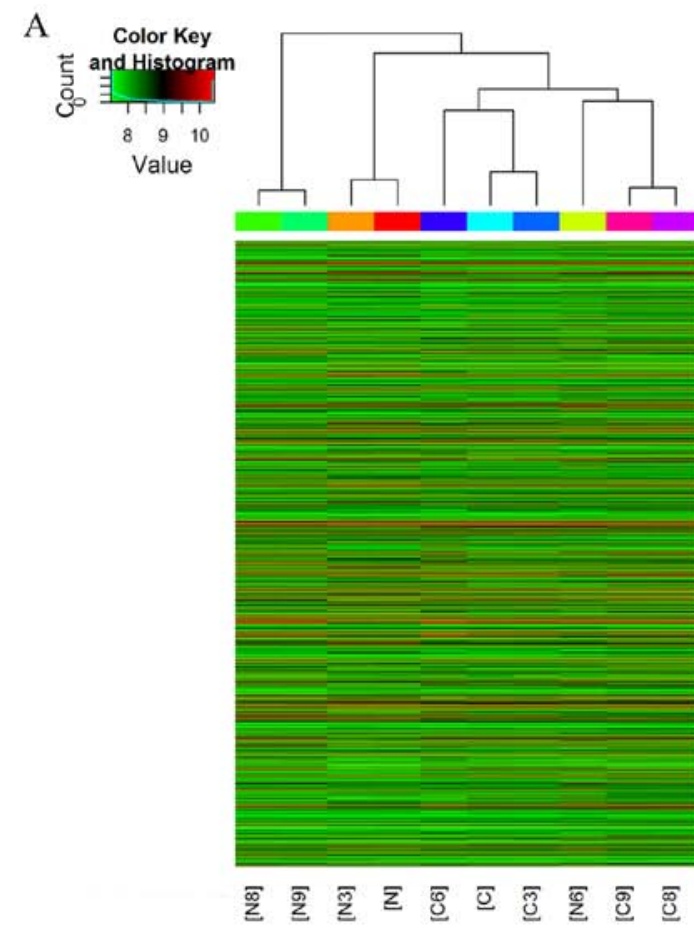

B
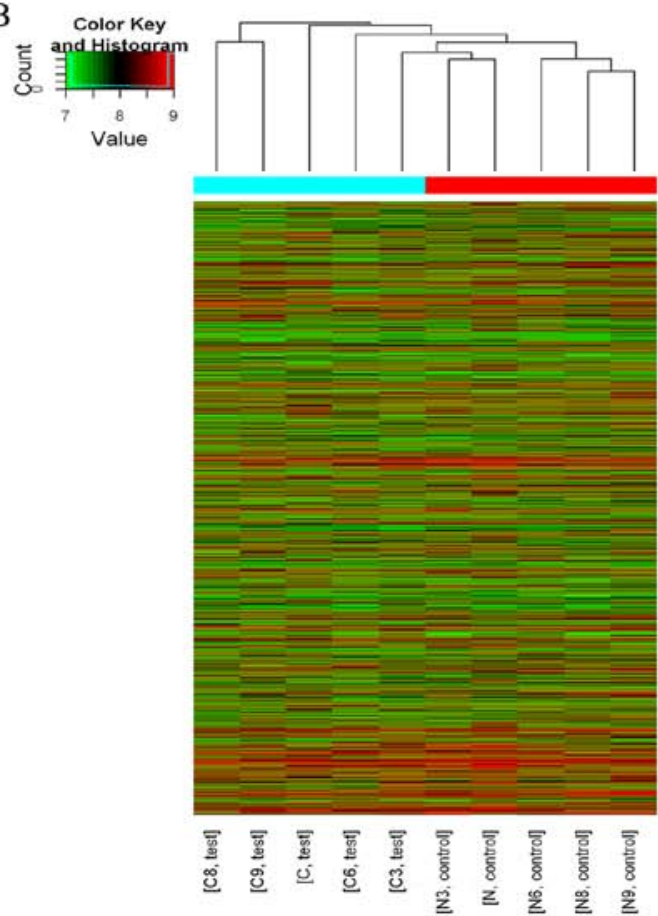
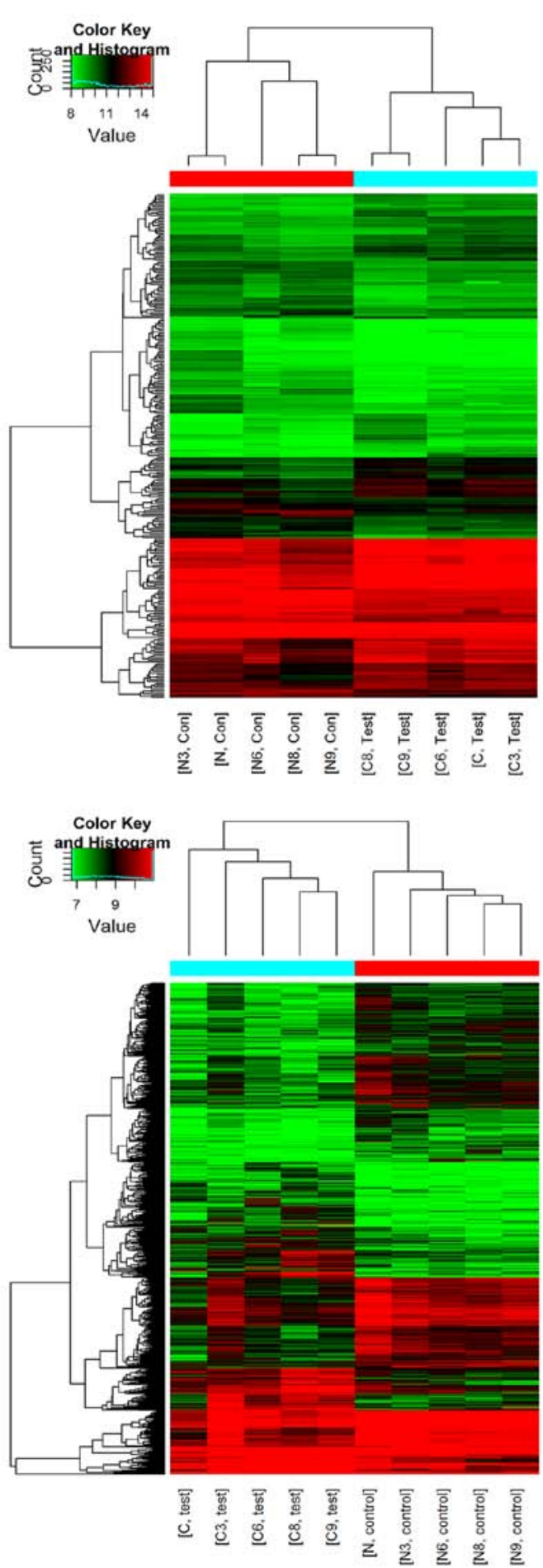

Figure 2. Heatmap showing the circRNA and mRNA expression profiles. Maps on the left are based on expression values of all circRNAs and mRNAs detected by microarray probes. Maps on the right correspond to normalized expression values of significantly changed circRNAs and mRNAs. The expression values are depicted in line with the color scale. The intensity increases from green to red. Each column represents one sample, and each row indicates a transcript. C, C3, C6, C8 and C9 represent cancer tissues, and N, N3, N6, N8 and N9 represent normal control tissues. (A) Hierarchical cluster analysis of all expressed circRNAs and dysregulated circRNAs with fold change $>1.5$ and $\mathrm{P}<0.05$. (B) Hierarchical cluster analysis of all expressed mRNAs and significantly changed mRNAs with fold change $>2.0$ and $\mathrm{P}<0.05$. circRNA, circular RNA.

constructed using fold change values (Fig. 1A and B), while the latter considered both fold change and statistical significance (Fig. 1C and D).

Hierarchical clustering analysis indicated that the circRNA and mRNA expression patterns were distinguishable between the groups (Fig. 2). The distribution of the dysregulated
circRNAs on human chromosomes was summarized, and the results revealed that these circRNAs were distributed among all chromosomes, apart from the Y sex chromosome (Fig. 3B). According to the associations with the protein-coding genes, the dysregulated circRNAs were classified into five categories: 214 were exonic, 35 were intronic, 7 were antisense, 3 were 

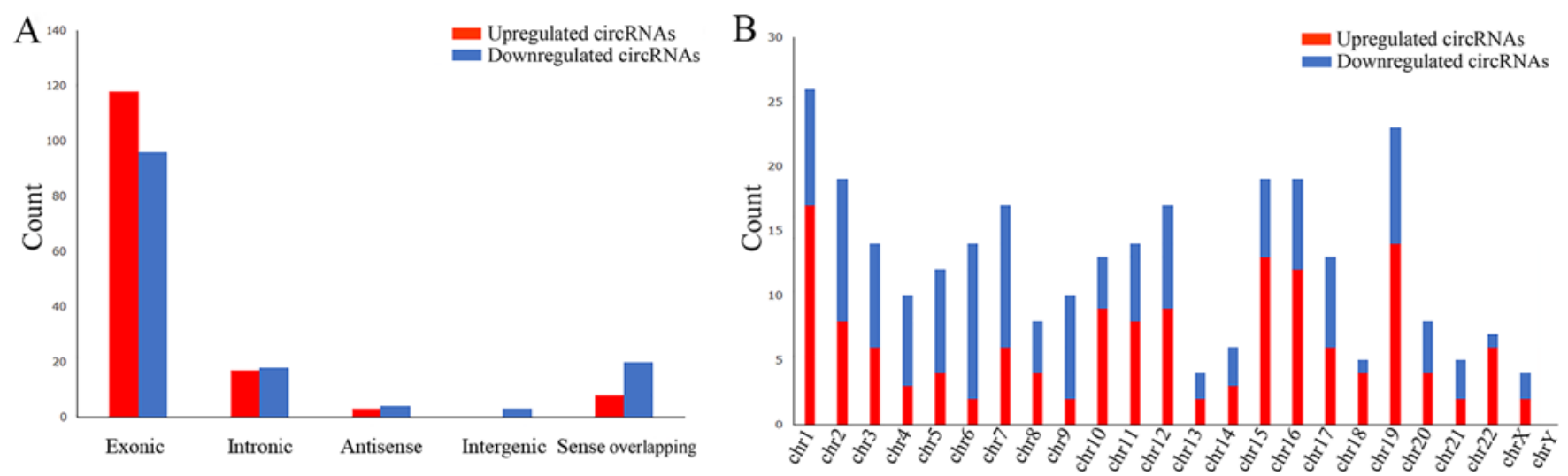

Figure 3. Characterizations of circRNAs in HNSCC. (A) Bar graph showing the types and counts of differentially regulated circRNAs. The circRNAs were classified into five types according to the relationship with their associated coding genes. (B) Distribution of differentially expressed circRNAs in human chromosomes. circRNA, circular RNA; HNSCC, head and neck squamous cell carcinoma.

A

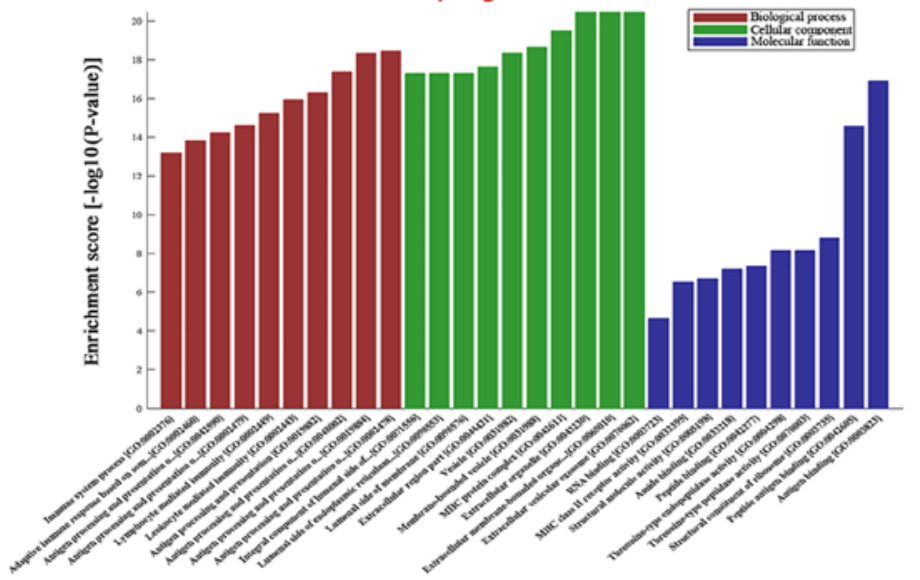

B

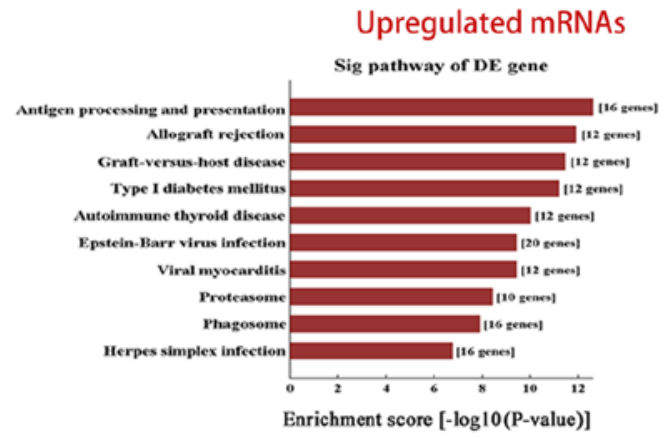

Downregulated mRNAs

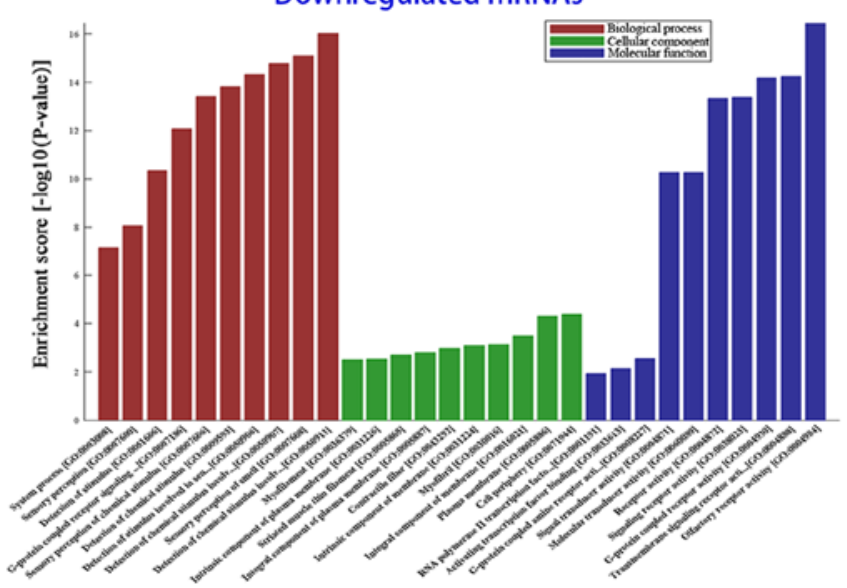

Downregulated mRNAs

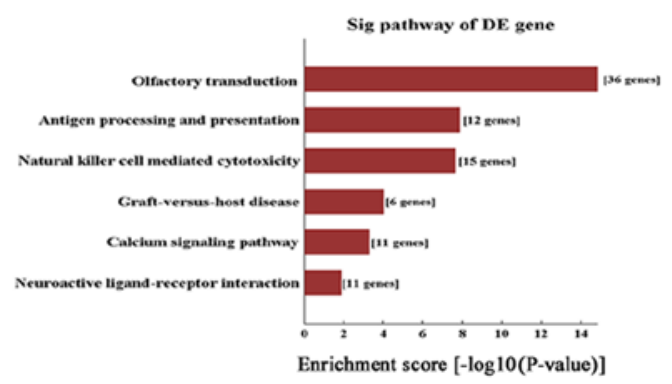

Figure 4. GO and pathway analyses. (A) GO annotation of up- and downregulated mRNAs with the top 10 enrichment scores, covering the domains of biological process, cellular component and molecular function, respectively. (B) KEGG pathway enrichment analysis of dysregulated mRNAs. GO, Gene Ontology; KEGG, Kyoto Encyclopedia of Genes and Genomes.

intergenic and 28 were sense overlapping (Fig. 3A). Thus, the expression profiles of the circRNAs and mRNAs in the HNSCC tissues were determined to differ from those in the matched tumor-adjacent tissues.

GO term and KEGG and pathway enrichment analyses. To date, the functions of the majority circRNAs have not been annotated. The functional prediction of circRNAs is largely based on the annotations of their interacting protein-coding genes. Therefore, the GO term and KEGG pathway enrichment analyses of dysregulated mRNAs can reveal the roles of significantly differentially expressed circRNAs, to a certain extent. According to the results of a GO term enrichment analysis, the principally enriched significant BP terms included immune system process, immune response and signaling transduction, such as 'antigen processing and presentation of exogenous peptide antigen' (GO:0002478), 'lymphocyte mediated immunity' (GO:0002449) and G-protein coupled receptor signaling pathway (GO:0007186). The most enriched CC terms were mostly regarding membranes, such 


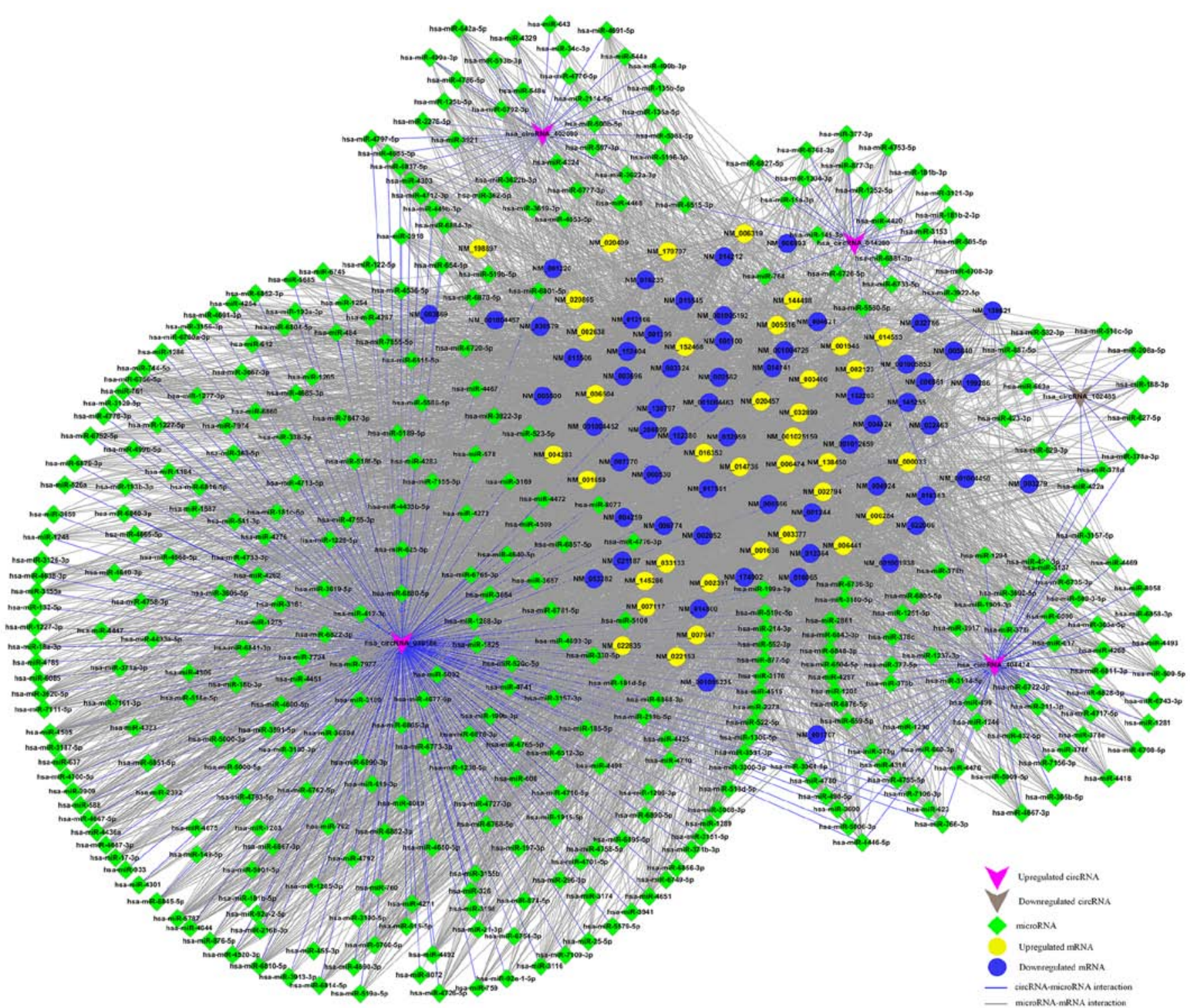

Figure 5. circRNA-miRNA-mRNA network. In this ceRNA network, each gene corresponds to a node, and 2 interacting genes based on seed sequence pairings are connected by a solid line. The ' $\mathrm{V}$ ' shapes represent circRNAs; the diamond shapes represent miRNAs; the ellipses represent mRNAs. The blue and gray lines represent circRNA-miRNA and miRNA-mRNA interactions, respectively. circRNA, circular RNA; miRNA, microRNA; ceRNA, competing endogenous RNA.

as 'integral component of membrane' (GO:0016021), 'plasma membrane' (GO:0005886) and 'extracellular membranebounded organelle' (GO:0065010). Furthermore, the most enriched MF terms were associated with receptor activity and receptor binding, including 'signaling receptor activity' (GO:0038023), 'signal transducer activity' (GO:0004871), 'activating transcription factor binding' (GO:0033613) and 'calcium-dependent protein binding' (GO:0048306). Fig. 4A shows the top 10 most significantly enriched GO terms in the $\mathrm{BP}, \mathrm{CC}$ and MF categories for the up- and downregulated mRNAs.

Pathway analysis was performed with the KEGG database. A total of 31 pathways associated with the upregulated mRNAs, and 6 related to the downregulated mRNAs were identified, including 'antigen processing and presentation' (hsa04612), 'natural killer cell mediated cytotoxicity' (hsa04650) and 'calcium signaling pathway' (hsa04020), among others. The most enriched pathways for the up- and downregulated protein-coding genes are listed in Fig. 4B. These biological processes, molecular functions and pathways likely contribute to the occurrence and development of HNSCC.

Construction of circRNA-miRNA-mRNA network. According to the ceRNA hypothesis, the members of each ceRNA group compete for the same MREs to regulate each other. We selected the top five upregulated and downregulated circRNAs as ranked by fold change from the microarray data (Table II), and then identified the potential ceRNA interactions via various bioinformatics methods. A summary of ceRNAs, including 5 circRNAs, 385 miRNAs and 5,148 mRNAs, was produced by merging the common targeting miRNAs. To further investigate the interactions between these ceRNAs in HNSCC tissues, we used only the differentially expressed mRNAs from the mRNA microarray data, and a specific ceRNA network, consisting of five circRNAs, 385 miRNAs and 96 mRNAs, for HNSCC was established (Fig. 5). 
Table II. Top 5 up- and downregulated circRNAs ranked by fold change $>3.0, \mathrm{P}<0.01$ and FDR $<0.05$ in the microarray data.

\begin{tabular}{|c|c|c|c|c|c|c|c|c|}
\hline circRNA & Regulation & $\begin{array}{l}\text { Absolute } \\
\text { fold change }\end{array}$ & P-value & FDR & Source & Alias & Chrom & $\begin{array}{l}\text { circRNA } \\
\text { type }\end{array}$ \\
\hline hsa_circRNA_0 & Up & 4.00 & $4.01 \mathrm{E}-05$ & 0.0173 & circBase & hsa_circ_0014280 & $\operatorname{chr} 1$ & Exonic \\
\hline hsa_circRNA_402089 & Up & 3.2867219 & $4.64 \mathrm{E}-05$ & 0.018507674 & 25242744 & - & chr19 & Exonic \\
\hline hsa_circRNA_036186 & Up & 3.2503065 & $7.05 \mathrm{E}-05$ & 0.022943867 & circBase & hsa_circ_0036186 & $\operatorname{chr} 15$ & Exonic \\
\hline hsa_circR & Up & 3.144 & $8.22 \mathrm{E}-05$ & 0.024789243 & 25070500 & _ & chr1 & Exonic \\
\hline hsa_circRNA_102485 & Down & 4.3172347 & $1.71 \mathrm{E}-05$ & 0.009591013 & circBase & hsa_circ_0050102 & chr19 & Exonic \\
\hline
\end{tabular}

'Regulation' describes that the experimental group has greater or lower intensity values than the control group. 'Absolute fold change' indicates the absolute ratio (no log scale) of normalized intensities between two conditions. 'P-value' indicates the P-value calculated from the paired t-test. 'FDR' indicates that FDR was calculated from the Benjamini-Hochberg false discovery rate. 'Alias' indicates the circRNA ID in circBase (http://circbase.mdc-berlin.de). 'circRNA type' indicates the circRNAs classified into 5 types (namely exonic, intronic, antisense, intergenic and sense overlapping. circRNA, circular RNA.

Table III. miRNAs associated with both tumor development and overall survival in the ceRNA network.

\begin{tabular}{|c|c|c|c|c|c|c|c|}
\hline \multirow[b]{2}{*}{ miRNA } & \multirow[b]{2}{*}{ Cancer } & \multicolumn{3}{|c|}{ Tumor development } & \multicolumn{3}{|c|}{ Tumor progression } \\
\hline & & t-test P-value & t-test FDR & Upregulated in: & Log-rank P-value & Log-rank FDR & Z-score \\
\hline hsa-miR-432-5p & HNSCC & $0.00177^{\mathrm{a}}$ & 0.00458 & Normal & $0.000457^{\mathrm{a}}$ & 0.143 & 3.496 \\
\hline hsa-miR-181c-5p & HNSCC & $0.00723^{\mathrm{a}}$ & 0.0166 & Normal & $0.00159^{\mathrm{a}}$ & 0.106 & 3.18 \\
\hline hsa-miR-377-5p & HNSCC & $0.000000119^{\mathrm{a}}$ & 0.000000861 & Normal & $0.00334^{\mathrm{a}}$ & 0.154 & 3.017 \\
\hline hsa-miR-125b-5p & HNSCC & $7.03 \mathrm{E}-11^{\mathrm{a}}$ & $1.23 \mathrm{E}-09$ & Normal & $0.00775^{\mathrm{a}}$ & 0.416 & 2.672 \\
\hline hsa-miR-193b-3p & HNSCC & $3.41 \mathrm{E}-13^{\mathrm{a}}$ & $1.47 \mathrm{E}-11$ & Tumor & $0.0285^{\mathrm{a}}$ & 0.686 & 2.163 \\
\hline hsa-miR-532-3p & HNSCC & $0.000438^{\mathrm{a}}$ & 0.00127 & Tumor & $0.0314^{\mathrm{a}}$ & 0.174 & 2.156 \\
\hline hsa-miR-629-3p & HNSCC & $2.38 \mathrm{E}-10^{\mathrm{a}}$ & 3.32E-09 & Tumor & $0.0389^{\mathrm{a}}$ & 0.183 & 2.059 \\
\hline
\end{tabular}

The Student's paired t-test was used to compare expression between tumor and normal tissues. Log-rank P-values and Z-scores obtained from univariate Cox proportional hazards analysis were used to show the relative effect of miRNA expression levels in regards to patient survival time. ${ }^{\mathrm{a}} \mathrm{P} \leq 0.05$.

Exploring miRNAs associated with tumor development and patient survival. miRNAs have been shown to play a crucial role in the pathogenesis and development of cancer (5-8). OncomiR online software (http://www.oncomir.org/) was used to systematically identify the miRNAs associated with development, staging and overall survival in 30 cancer types, and these data were used to identify the miRNAs associated with tumor formation and patient survival in HNSCC. The results revealed that there were 376 miRNAs associated with tumor development and 172 miRNAs related to tumor prognosis in HNSCC. Among these, 80 overlapping miRNAs were found in both the tumor development and prognosis groups, suggesting that these miRNAs were likely to be involved in the regulation of both HNSCC occurrence and metastasis. Finally, we retained the miRNAs associated with both tumor development and patient survival from the ceRNA network (Fig. 6A and Table III), and thus constructed a network consisting of 4 circRNAs, 8 miRNAs and 51 mRNAs (Fig. 6B).

Prediction of responsible and functional miRNA-mRNA axes. To explore the molecular mechanisms of action of
circRNAs in HNSCC, we predicted the circRNA-miRNA axes associated with cancer-related pathways. DIANAmiRPath v3.0 allows the analysis of miRNA-associated pathways as well as the prediction of miRNA targets using DIANA-microT-CDS v5.0 and/or DIANA-TarBase v7.0. We queried with the 8 miRNAs from our network, including miR-377-5p, -432-5p, -125b-5p, -629-3p, -532-3p, $-181 c-5 p,-193 b-3 p$ and $-135 b-5 p$, in DIANA-miRPath v3.0DIANA-TarBase v7.0 and microT-CDS v5.0 to predict the potential target genes and pathways, with thresholds of $\mathrm{P}<0.05$ and MicroT score $>0.8$. The overlapping results from DIANA-TarBase v7.0 and DIANA-microT-CDS v5.0, and the aforementioned analyses on ceRNAs revealed that miR-193b-3p was matched with 14-3-3 $\zeta$ according to the results of all three methods, indicating that miR-193b-3p had a significant potential to interact with 14-3-3ל (Fig. 7A). Therefore, 14-3-3 $\zeta$ and miR-193b-3p were analyzed, and the results from pathway intersection analysis indicated that miR-193b-3p was likely to participate in the Hippo signaling pathway (hsa04390) $(\mathrm{P}=5.442667 \mathrm{e}-06)$ by binding to $14-3-3 \zeta$ in HNSCC (Fig. 7B). 
A

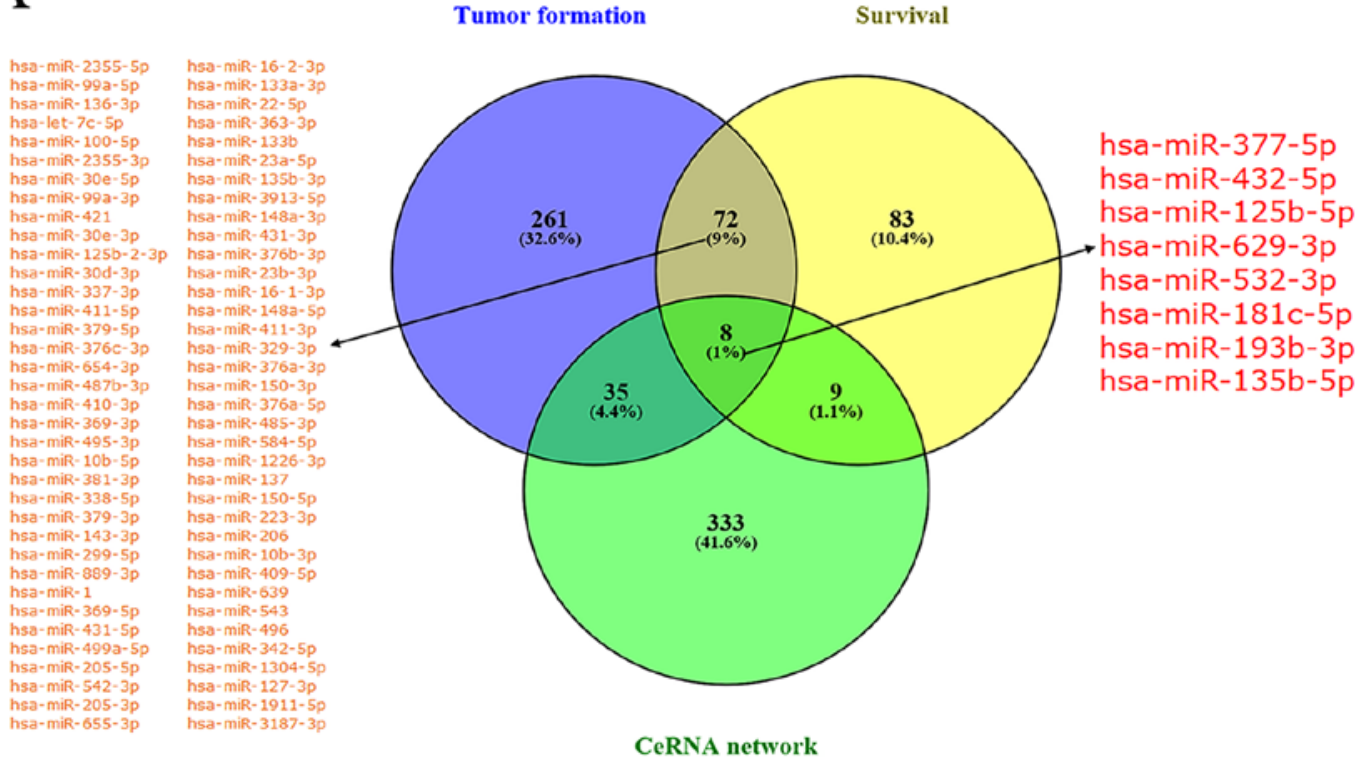

B

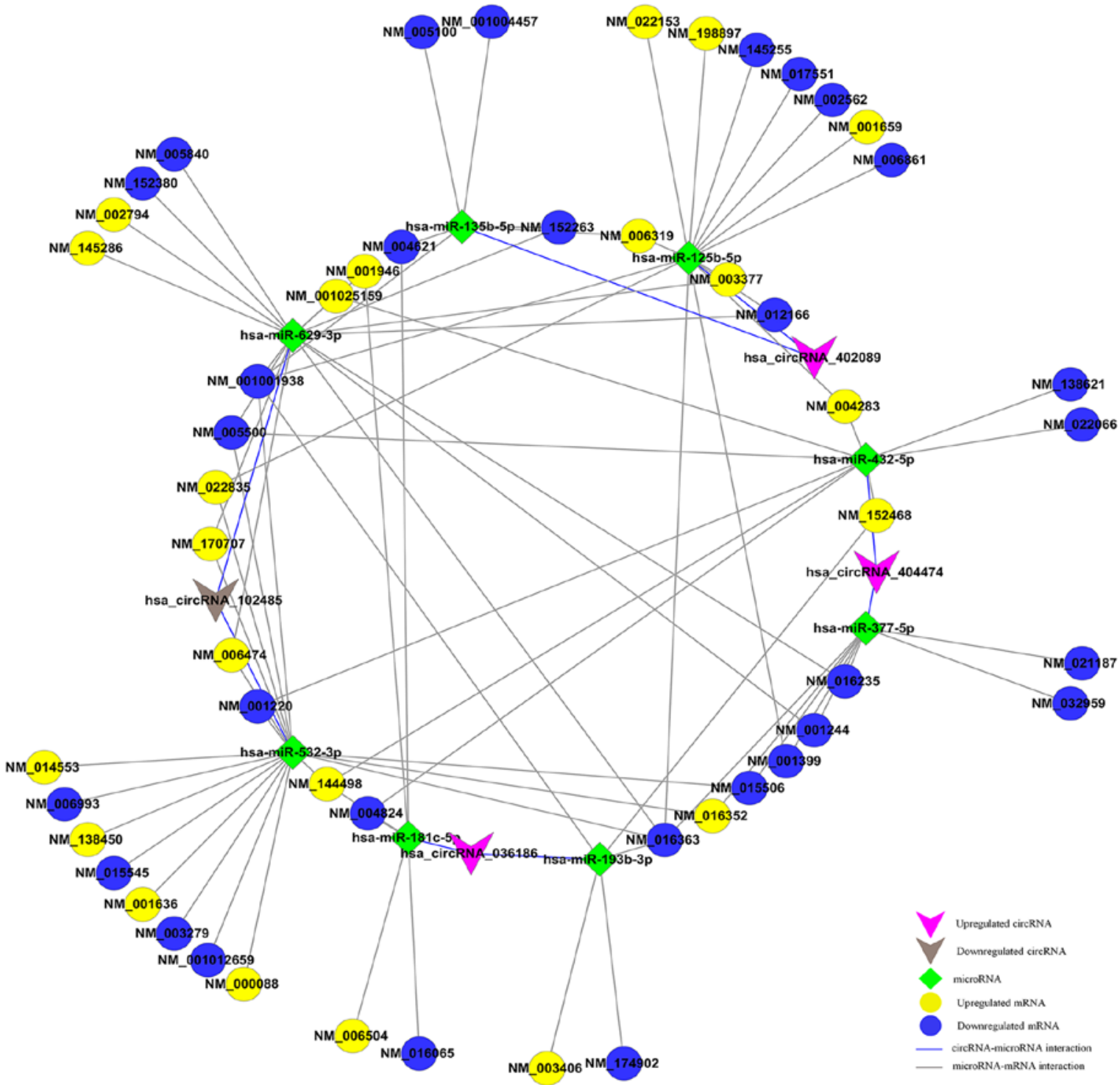

Figure 6. miRNAs associated with tumor development and patient survival. (A) Venn diagram indicating that the predicted miRNAs derived from the results of OncomiR and ceRNA analyses. miR-377-5p, miR-432-5p, miR-125b-5p, miR-629-3p, miR-532-3p, miR-181c-5p, miR-193b-3p and miR-135b-5p were intersecting. (B) The circRNA-miRNA-mRNA network consists of 4 circRNAs, 8 microRNAs and 51 mRNAs. circRNA, circular RNA; miRNA, microRNA; ceRNA, competing endogenous RNA. 
A
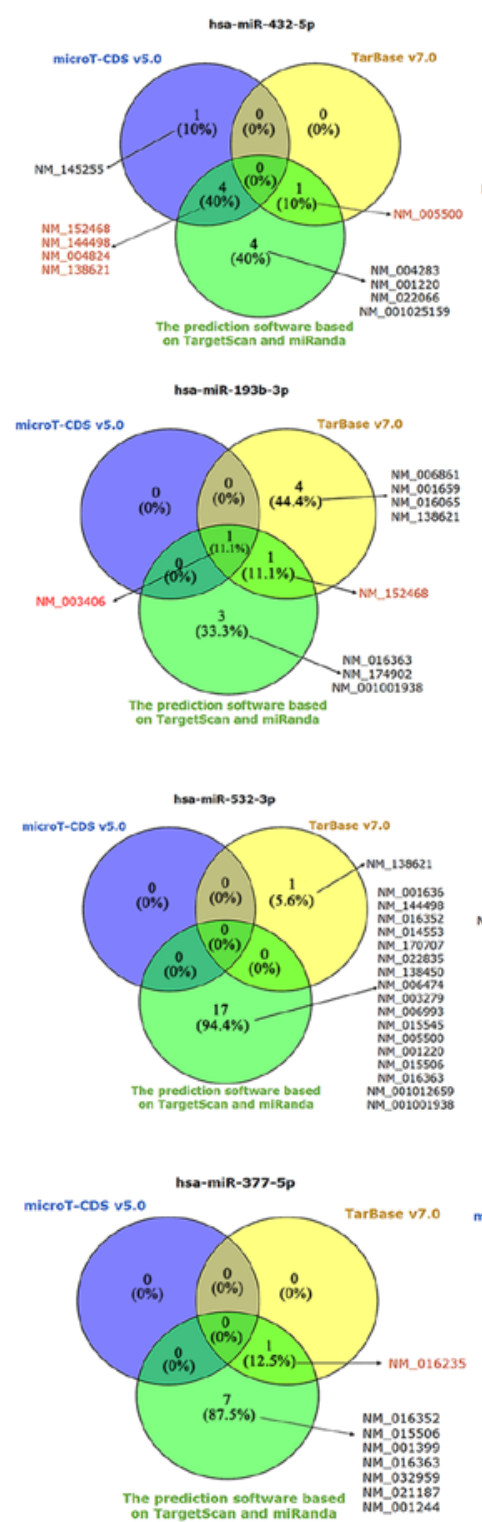
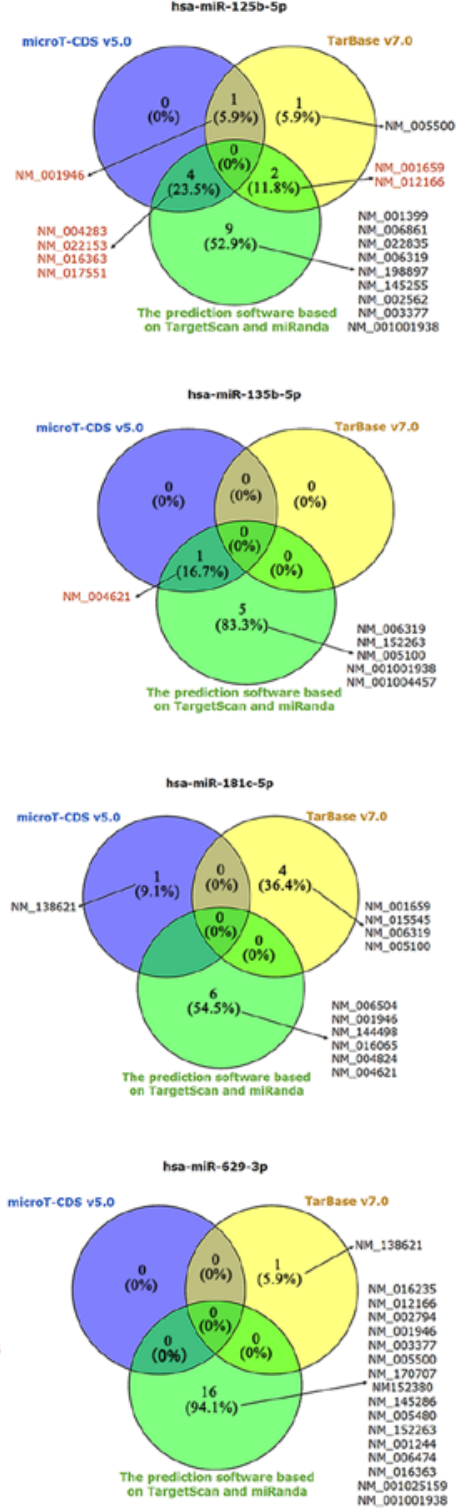

$\mathrm{B}$

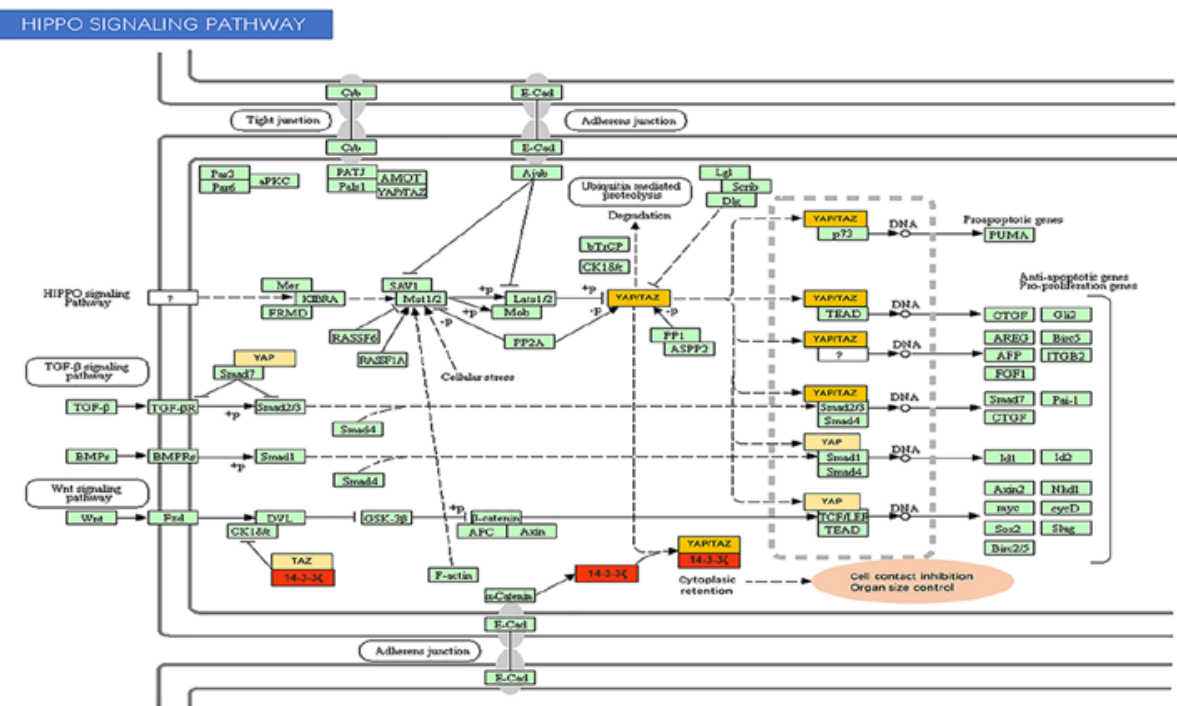

Figure 7. miRNA target prediction and miRNA-mediated signaling pathways. (A) Venn diagrams showing the target genes of miRNAs predicted by DIANAmicroT-CDS v5.0, DIANA-TarBase v7.0 and miRNA target prediction software based on TargetScan and miRanda. The results indicated that NM_003406 (red) was targeted by miR-193b-3p is intersected. (B) Mapping of the Hippo signaling pathway indicated that 14-3-3६ (red) mediated by miR-193b-3p serves as a crucial factor significantly engaged in the function of cell-contact inhibition and organ size control by combining with yes-associated protein $1 / \mathrm{WW}$ domain-containing transcription regulator protein 1 (YAP/TAZ). miRNA, microRNA. 
B
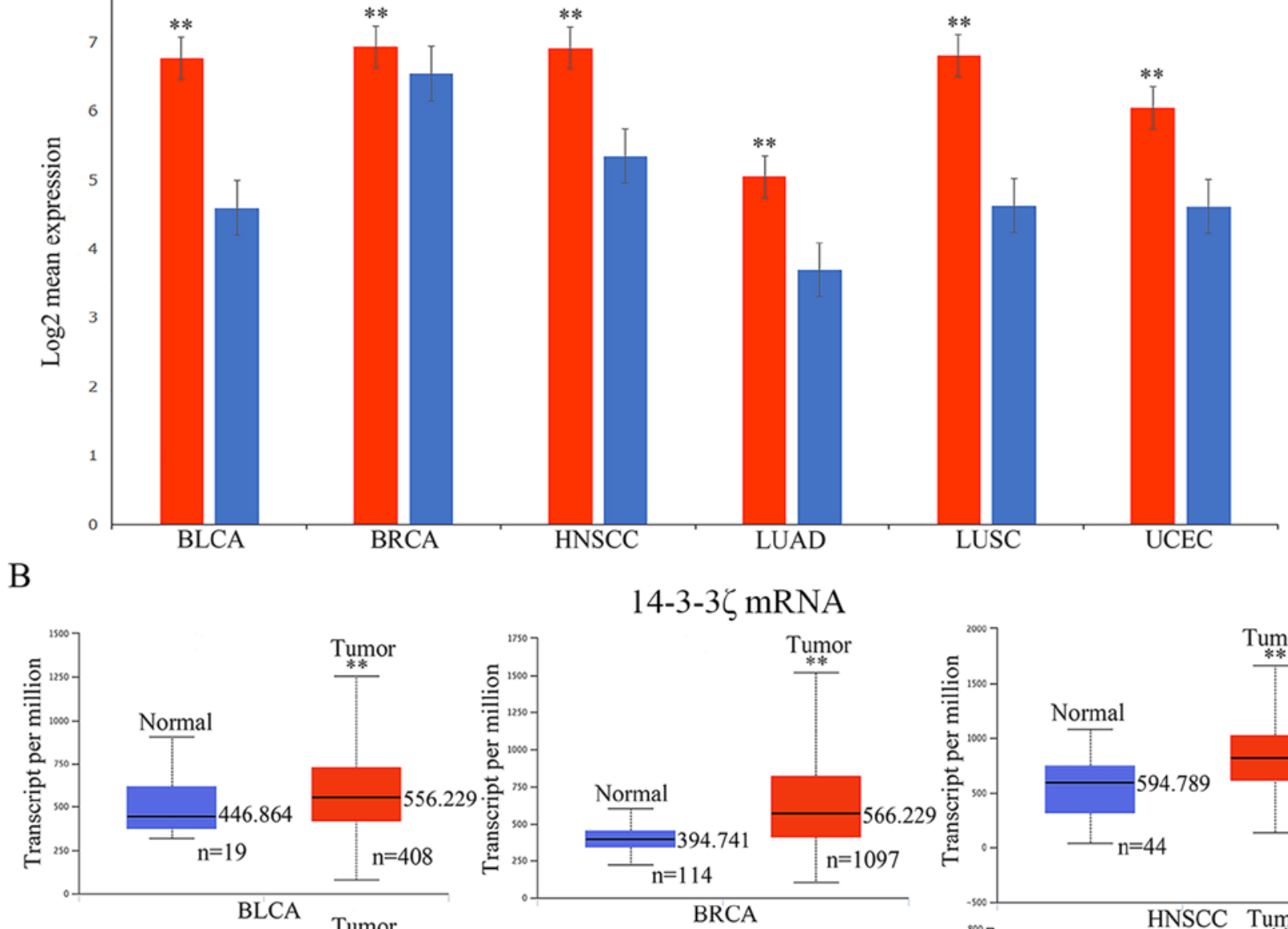

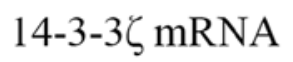
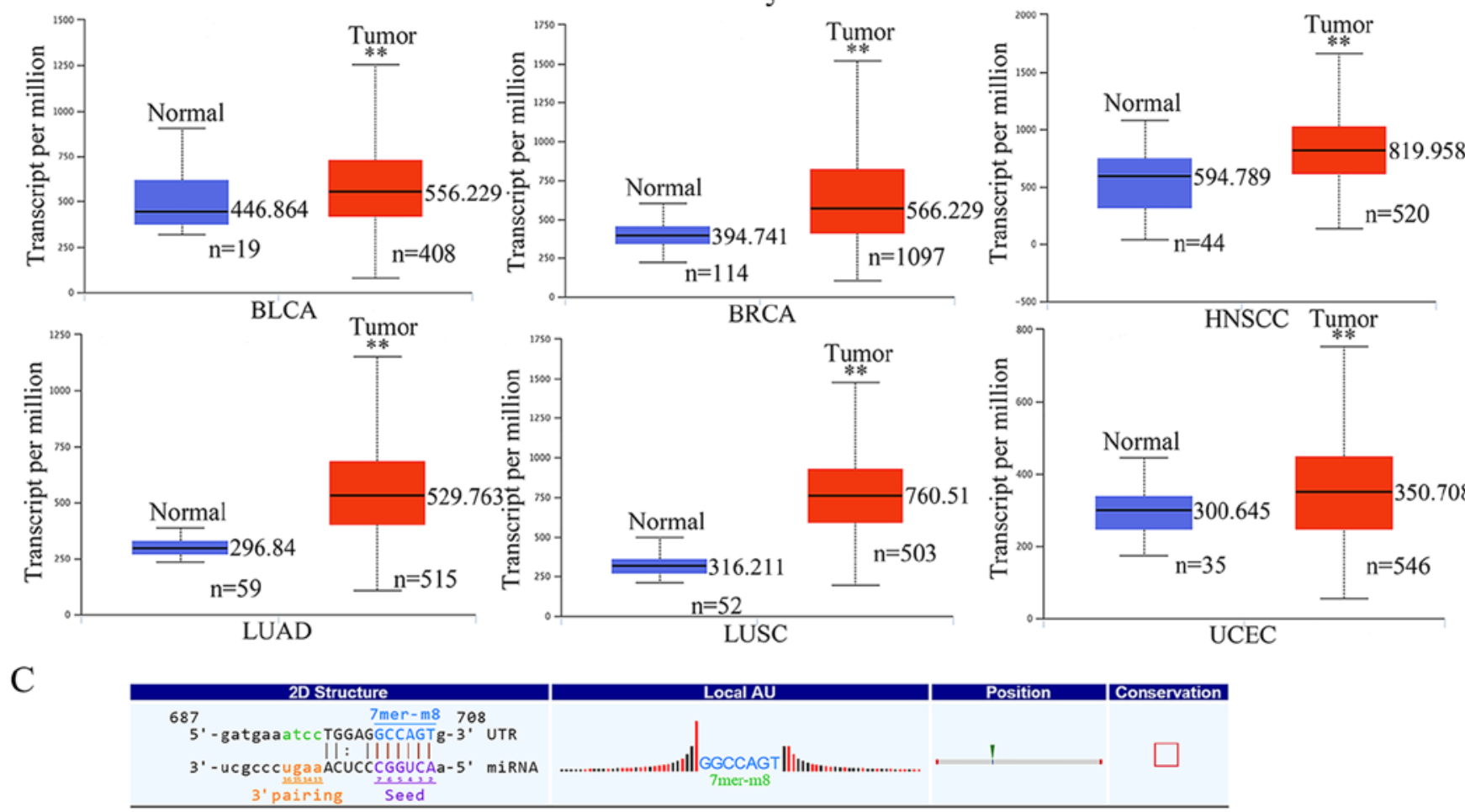

Figure 8. Expression changes of RNA transcripts and circRNA-miRNA interaction. (A) Bar graph showing the log2 transform of the mean miR-193b-3p expression in both tumor (red) and non-tumor tissues (blue). ${ }^{* *} \mathrm{P}<0.01$. (B) Boxplot showing relative expression of $14-3-3 \zeta$ in normal and cancer samples. ${ }^{* *} \mathrm{P}<0.01$. (C) The interaction of circRNA_036186-miR-193b-3p predicted based on data from TargetScan and miRanda. BRCA, breast invasive carcinoma; HNSCC, head and neck squamous cell carcinoma; LUAD, lung adenocarcinoma; LUSC, lung squamouscell carcinoma; UCEC, uterine corpus endometrial carcinoma.

Prediction of the potential function of circRNA_036186

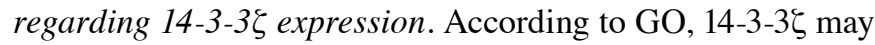
act through 'protein targeting' (GO:0006605), 'anti-apoptosis' (GO:0006916) and 'signal transduction' (GO:0007165). All these GO biological processes are associated with the occurrence and development of cancer. We analyzed the expression differences for miR-193b-3p and 14-3-3ל in malignant tumors using OncomiR and UALCAN, respectively. The results revealed that the expression levels of miR-193b-3p and 14-3-3̧ were both upregulated $(\mathrm{P}<0.01)$ in the six malignant tumor samples compared with in the adjacent tissues (Fig. 8A and B). However, evidence has indicated that the mRNA expression level is negatively associated with its corresponding miRNA (4), and we hypothesized that this effect may be attributed to the participation of circRNA expression. As a miRNA sponge, circRNAs can counteract, and even reverse, the functional 
Table IV. Microarray and RT-qPCR analyses of selected circRNAs and mRNAs.

\begin{tabular}{lcccccccc}
\hline & \multicolumn{3}{c}{ hsa_circRNA_036186 } & & \multicolumn{3}{c}{$14-3-3 \zeta$} \\
\cline { 2 - 3 } Detection method & Absolute fold change & Regulation & P-value & & Absolute fold change & Regulation & P-value \\
\hline Microarray & 3.250307 & $\mathrm{Up}$ & $0.000071^{\mathrm{a}}$ & & 2.318862 & & $\mathrm{Up}$ & $0.021603^{\mathrm{a}}$ \\
RT-qPCR & 2.836309 & $\mathrm{Up}$ & $0.001582^{\mathrm{a}}$ & & 1.7610711 & & $\mathrm{Up}$ & $0.011234^{\mathrm{a}}$ \\
\hline
\end{tabular}

The expression levels of circRNA_036186 and 14-3-3̧ were determined by RT-qPCR. Each RT-qPCR assay was performed at least three times. The RT-qPCR and microarray data were in accord. ${ }^{a} \mathrm{P}<0.05$. circRNA, circular RNA.

differences caused by an alteration in miRNA expression. In this study, according to the results of ceRNA analysis, there was an evolutionarily conserved regulatory region (7mer-m8) in circRNA_036186 for binding to miR-193b-3p (Fig. 8C), and the expression of circRNA_036186 was significantly higher in the HNSCC tumor tissues compared with the para-carcinoma tissues in the microarray data. Therefore, we proposed that the circRNA_036186-miR-193b-3p-14-3-3 $\zeta$ axis was likely crucial in the occurrence and prognosis of a variety of cancer types, including HNSCC. Furthermore, our results from microarray analysis revealed that the level of $14-3-3 \xi$ was upregulated in HNSCC, compared with the control, which was consistent with the data from TCGA (36). This indicated that circRNA_036186 probably serves as a crucial and positive regulator of $14-3-3 \zeta$ and is significantly engaged in the Hippo signaling pathway in HNSCC.

Validation of the differential expression levels of circRNA_036186 and 14-3-3\}. The results of microarray analysis of circRNA_036186 and 14-3-3 $\zeta$ were verified by RT-qPCR with the same RNA samples used for the microarray analysis. The results of RT-qPCR assay revealed that the expression levels of circRNA_036186 and 14-3-3६ were each upregulated in HNSCC compared with the controls. The results are consistent with those of the microarray assay (Table IV); therefore, the RT-qPCR data verified the accuracy of the microarray results. These results provide evidence for our hypothesis that circRNA_036186 participates in the pathogenesis and development of HNSCC.

\section{Discussion}

As a group of functional molecules with abundant biological information, RNAs have particular value in the prevention and treatment of human diseases. In eukaryotic cells, protein coding RNA accounts for only $2 \%$ of the genome, and the vast majority of transcripts are ncRNAs (43). In recent years, the biogenesis and functions of ncRNAs have attracted increasing attention (44). The malignant behaviors of cancer cells are usually regulated by multiple gene products, and, theoretically, these genes can be grouped into 'networks' based on their interactions $(10,33,45,46)$. These interactions may provide new insight into the potential mechanisms of neoplasia and lay the groundwork for a new approach to cancer treatment.

miRNAs are the most well-studied ncRNAs, and are a class of small ncRNAs $\sim 22$ nucleotides (nt) in length that, by binding to MREs, can block protein translation or modulate mRNA stability on a post-transcriptional level $(4,47,48)$. Unlike miRNAs, the functions and regulatory mechanisms of circRNAs remain largely unknown. Fortunately, the application of circRNA microarray techniques has allowed for the identification of differentially expressed circRNAs and the exploration of their functions (49). An increasing number of circRNAs have been found to be cell type- or tissue-specific, rendering them more predictive of the potential biological functions $(50,51)$. Evidence has indicated that the functions of circRNAs stem from three main aspects: Absorbing miRNA sponge $(24,29,30,52)$, binding RNA-binding proteins (RBPs) (16) and translating peptides $(53,54)$. To date, the miRNA sponge theory remains the most popular hypothesis.

HNSCC is an aggressive tumor that often occurs with recurrence and metastasis, and the survival rate has changed little in recent decades. Over the past few years, an increasing number of experimental and clinical studies have suggested that ncRNAs play an important role in HNSCC pathophysiology (55 and refs. therein). For instance, miR-150-5p and miR-150-3p, antitumor miRNAs, have been shown to significantly inhibit cell aggressiveness by directly targeting SPARC (osteonectin), cwcv and kazal like domains proteoglycan 1 (SPOCK1) in HNSCC (56). miR-206 inhibited cell proliferation by arresting the cell cycle in S-phase and targeting histone deacetylase 6 (HDAC6) via the phosphatase and tensin homolog (PTEN)/ AKT/mammalian target of rapamycin (mTOR) pathway (57). Additionally, by evaluating the associations between miRNA polymorphisms and HNSCC risk according to the cancer site, miR-605 rs2043556 and miR-196a2 rs11614913 have been shown as likely to affect genetic susceptibility to oral squamous cell carcinoma (58). Evidence has suggested that circRNA_100290 may serve as a sponge of the miR-29 family to regulate cyclin-dependent kinase 6 (CDK6) expression in oral squamous cell carcinoma (59).

In the present study, microarray screening and RT-qPCR verification were used to identify circRNA and mRNA expression profiles, and ceRNA analysis was performed to evaluate the circRNA-miRNA-mRNA interactions. The results of microarray analysis and RT-qPCR revealed that circRNA_036186 and 14-3-3 $\zeta$ were highly expressed in HNSCC compared with the controls. Using bioinformatics analysis, we found that circRNA_036186 and 14-3-3ל had the same seed sequence binding site to for interaction with miR-193b-3p. It has been previously reported that miR-193b-3p and $14-3-3 \zeta$ are closely related to the occurrence and prognosis 
of cancer (60-64). Existing evidence for miR-193b-3p has been largely focused on the negative regulation of the expression of oncogenes in cancer development (65-67), and the effect on carcinogenesis and prognosis is the most well-characterized

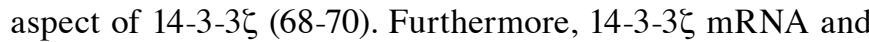
protein expression have been reported to be upregulated in HNSCC tissue samples, and the overexpression of 14-3-3 $\zeta$ promotes cell growth, as well as morphological changes. By contrast, reduced 14-3-3 $\zeta$ levels increase the relative proportion of cells in the G1/G0-phase (71).

The results from DIANA-miRPath v3.0 revealed that miR-193b-3p significantly engaged in the Hippo signaling pathway via 14-3-3 $\zeta$ with the function of cell contact inhibition and organ size control by combining with yes-associated protein 1 (YAP)/WW domain-containing transcription regulator protein 1 (TAZ). A growing body of evidence has suggested that the Hippo pathway may act as a critical role in organ size control by promoting cell growth, cell proliferation and preventing apoptosis (72-75). A recent study reported that the interaction between 14-3-3 $\zeta$ and YAP, as a negative regulatory feedback loop, may play an important role in cell proliferation and survival in gastric cancer (76). Moreover, using TCGA data, we identified a group of miRNAs that were relevant to both cancer development and patient survival in HNSCC. The result revealed that the expression of miR-193b-3p in HNSCC tissues was significantly higher than that in the adjacent tissues. However, to date, at least to the best of our knowledge, there have been no reports on the functions and mechanisms of the circRNA_036186-miR-193b-3p-14-3-3 pathway in patients with HNSCC. Considering the data from previous studies in addition to the results of the current study, the emerging endogenous circRNA molecule circRNA_036186 probably serves as a crucial regulator of $14-3-3 \zeta$ by acting as a miRNA sponge to inhibit miR-193b-3p function, and hence participates in the Hippo signaling pathway to exert an effect on the course of HNSCC.

In this study, due to the limited number of available tissues samples, we only analyzed circRNA and mRNA expression profiles in five paired HNSCC tissues and their normal controls. More samples should be obtained and analyzed in the future to verify our results. Additionally, our results were mainly based on bioinformatics predictions; whether they can be extended to wider applications remains undefined. The main limitation of the present study was that at present, there is no convincing functional study to verify that circRNA_036186 inhibited miR-193b-3p to result in regulation of the Hippo signaling pathway; further functional studies to verify the roles of circRNA_036186 are required.

In conclusion, the findings of this study revealed the circRNA and mRNA expression characteristics in HNSCC and highlighted the possibility that circRNA-036186 may serve as a diagnostic and therapeutic biomarker in HNSCC.

\section{Acknowledgements}

Not applicable.

\section{Funding}

This study was supported by grants from the National Natural Science Foundation of China (no. 81372877), the National
Young Scholars Science Foundation of China (no. 81102058), the Foundation of Education Bureau of Liaoning Province (no. L2014317), the Natural Science Foundation of Liaoning Province (no. 2014021096), the Excellent Talent Fund Project of Higher Education of Liaoning Province (no. LJQ2014087), the Doctoral Scientific Research Foundation of Liaoning Province (no. 201501002), and the Young and Middle-aged Science and Technology Innovation Talent Support Project of Shenyang (no. RC170489).

\section{Availability of data and materials}

The datasets used and/or analyzed during the current study are available from the corresponding author on reasonable request.

\section{Authors' contributions}

WLW, CFS and FYL conceived and designed the experiments. ZY and YJZ carried out the experiments. WLW analyzed the data, prepared the figures and wrote the manuscript. PL and YKN were mainly involved in the collection and pathological examinations of patient specimens. CFS supervised the whole project. All authors have read and approved the final manuscript.

\section{Ethics approval and consent to participate}

This study was approved and supervised by The Ethics Committee of China Medical University (Shenyang, China). Written informed consent was obtained from all patients.

\section{Patient consent for publication}

Not applicable.

\section{Competing interests}

The authors declare that they have no competing interests.

\section{References}

1. Marur S and Forastiere AA: Head and neck squamous cell carcinoma: Update on epidemiology, diagnosis, and treatment. Mayo Clin Proc 91: 386-396, 2016.

2. Siegel RL, Miller KD and Jemal A: Cancer statistics, 2016. CA Cancer J Clin 66: 7-30, 2016.

3. Chen W, Zheng R, Baade PD, Zhang S, Zeng H, Bray F, Jemal A, Yu XQ and He J: Cancer statistics in China, 2015. CA Cancer J Clin 66: 115-132, 2016.

4. Fabian MR, Sonenberg N and Filipowicz W: Regulation of mRNA translation and stability by microRNAs. Annu Rev Biochem 79: 351-379, 2010

5. Li H, Xie S, Liu M, Chen Z, Liu X, Wang L, Li D and Zhou Y: The clinical significance of downregulation of mir-124-3p, mir-146a-5p, mir-155-5p and mir-335-5p in gastric cancer tumorigenesis. Int J Oncol 45: 197-208, 2014.

6. Cao J, Cai J, Huang D, Han Q, Yang Q, Li T, Ding H and Wang Z: miR-335 represents an invasion suppressor gene in ovarian cancer by targeting Bcl-w. Oncol Rep 30: 701-706, 2013.

7. Croce CM: Causes and consequences of microRNA dysregulation in cancer. Nat Rev Genet 10: 704-714, 2009.

8. Esquela-Kerscher A and Slack FJ: Oncomirs - microRNAs with a role in cancer. Nat Rev Cancer 6: 259-269, 2006.

9. Poliseno L, Salmena L, Zhang J, Carver B, Haveman WJ and Pandolfi PP: A coding-independent function of gene and pseudogene mRNAs regulates tumour biology. Nature 465: $1033-1038,2010$ 
10. Salmena L, Poliseno L, Tay Y, Kats L and Pandolfi PP: A ceRNA hypothesis: The Rosetta Stone of a hidden RNA language? Cell 146: 353-358, 2011.

11. Rybak-Wolf A, Stottmeister C, Glažar P, Jens M, Pino N, Giusti S, Hanan M, Behm M, Bartok O, Ashwal-Fluss R, et al: Circular RNAs in the mammalian brain are highly abundant, conserved, and dynamically expressed. Mol Cell 58: 870-885, 2015.

12. Qu S, Yang X, Li X, Wang J, Gao Y, Shang R, Sun W, Dou K and Li H: Circular RNA: A new star of noncoding RNAs. Cancer Lett 365: 141-148, 2015.

13. Jeck WR, Sorrentino JA, Wang K, Slevin MK, Burd CE, Liu J, Marzluff WF and Sharpless NE: Circular RNAs are abundant, conserved, and associated with ALU repeats. RNA 19: 141-157, 2013.

14. Zhang Y, Zhang XO, Chen T, Xiang JF, Yin QF, Xing YH, Zhu S Yang $\mathrm{L}$ and Chen LL: Circular intronic long noncoding RNAs. Mol Cell 51: 792-806, 2013.

15. Shen T, Han M, Wei G and Ni T: An intriguing RNA species - perspectives of circularized RNA. Protein Cell 6: 871-880, 2015.

16. Li Z, Huang C, Bao C, Chen L, Lin M, Wang X, Zhong G, $\mathrm{Yu} \mathrm{B}, \mathrm{Hu}$ W, Dai L, et al: Exon-intron circular RNAs regulate transcription in the nucleus. Nat Struct Mol Biol 22: 256-264, 2015.

17. Chen LL: The biogenesis and emerging roles of circular RNAs. Nat Rev Mol Cell Biol 17: 205-211, 2016.

18. Ashwal-Fluss R, Meyer M, Pamudurti NR, Ivanov A, Bartok O, Hanan M, Evantal N, Memczak S, Rajewsky N and Kadener S: circRNA biogenesis competes with pre-mRNA splicing. Mol Cell 56: 55-66, 2014.

19. Wilusz JE and Sharp PA: Molecular biology. A circuitous route to noncoding RNA. Science 340: 440-441, 2013.

20. Vicens Q and Westhof E: Biogenesis of circular RNAs. Cell 159: 13-14, 2014.

21. Zhang XO, Wang HB, Zhang Y, Lu X, Chen LL and Yang L: Complementary sequence-mediated exon circularization. Cell 159: 134-147, 2014.

22. Westholm JO, Miura P, Olson S, Shenker S, Joseph B, Sanfilippo P, Celniker SE, Graveley BR and Lai EC: Genome-wide analysis of drosophila circular RNAs reveals their structural and sequence properties and age-dependent neural accumulation. Cell Reports 9: 1966-1980, 2014.

23. Suzuki H, Aoki Y, Kameyama T, Saito T, Masuda S, Tanihata J, Nagata T, Mayeda A, Takeda S and Tsukahara T: Endogenous multiple exon skipping and back-splicing at the DMD mutation hotspot. Int J Mol Sci 17: 17, 2016.

24. Hansen TB, Jensen TI, Clausen BH, Bramsen JB, Finsen B, Damgaard CK and Kjems J: Natural RNA circles function as efficient microRNA sponges. Nature 495: 384-388, 2013.

25. Zheng Q, Bao C, Guo W, Li S, Chen J, Chen B, Luo Y, Lyu D, Li Y, Shi G, et al: Circular RNA profiling reveals an abundant circHIPK 3 that regulates cell growth by sponging multiple miRNAs. Nat Commun 7: 11215, 2016.

26. Guo JU, Agarwal V, Guo H and Bartel DP: Expanded identification and characterization of mammalian circular RNAs. Genome Biol 15: 409, 2014.

27. Tay Y, Rinn J and Pandolfi PP: The multilayered complexity of ceRNA crosstalk and competition. Nature 505: 344-352, 2014

28. Ghosal S, Das S, Sen R, Basak P and Chakrabarti J: Circ2Traits: A comprehensive database for circular RNA potentially associated with disease and traits. Front Genet 4: 283, 2013.

29. Lukiw WJ: Circular RNA (circRNA) in Alzheimer's disease (AD). Front Genet 4: 307, 2013

30. Hansen TB, Kjems J and Damgaard CK: Circular RNA and miR-7 in cancer. Cancer Res 73: 5609-5612, 2013.

31. Enright AJ, John B, Gaul U, Tuschl T, Sander C and Marks DS MicroRNA targets in Drosophila. Genome Biol 5: R1, 2003.

32. Pasquinelli AE: MicroRNAs and their targets: Recognition, regulation and an emerging reciprocal relationship. Nat Rev Genet 13: 271-282, 2012

33. Assenov Y, Ramírez F, Schelhorn SE, Lengauer T and Albrecht M: Computing topological parameters of biological networks. Bioinformatics 24: 282-284, 2008.

34. Shannon P, Markiel A, Ozier O, Baliga NS, Wang JT, Ramage D, Amin N, Schwikowski B and Ideker T: Cytoscape: A software environment for integrated models of biomolecular interaction networks. Genome Res 13: 2498-2504, 2003.

35. Wong NW, Chen Y, Chen S and Wang X: OncomiR: An online resource for exploring pan-cancer microRNA dysregulation. Bioinformatics 34: 713-715, 2018.
36. Chandrashekar DS, Bashel B, Balasubramanya SAH, Creighton CJ Ponce-Rodriguez I, Chakravarthi BVSK and Varambally S: UALCAN: A portal for facilitating tumor subgroup gene expression and survival analyses. Neoplasia 19: 649-658, 2017.

37. Vlachos IS, Zagganas K, Paraskevopoulou MD, Georgakilas G, Karagkouni D, Vergoulis T, Dalamagas T and Hatzigeorgiou AG: DIANA-miRPath v3.0: Deciphering microRNA function with experimental support. Nucleic Acids Res 43W1: W460-W466, 2015.

38. Li JH, Liu S, Zhou H, Qu LH and Yang JH: starBase v2.0: Decoding miRNA-ceRNA, miRNA-ncRNA and protein-RNA interaction networks from large-scale CLIP-Seq data. Nucleic Acids Res 42D1: D92-D97, 2014.

39. Vlachos IS, Paraskevopoulou MD, Karagkouni D, Georgakilas G, Vergoulis T, Kanellos I, Anastasopoulos IL, Maniou S, Karathanou K, Kalfakakou D, et al: DIANA-TarBase v7.0: indexing more than half a million experimentally supported miRNA:mRNA interactions. Nucleic Acids Res 43D: D153-D159, 2015.

40. Paraskevopoulou MD, Georgakilas G, Kostoulas N, Vlachos IS, Vergoulis T, Reczko M, Filippidis C, Dalamagas T and Hatzigeorgiou AG: DIANA-microT web server v5.0: Service integration into miRNA functional analysis workflows. Nucleic Acids Res 41W: W169-W173, 2013.

41. Reczko M, Maragkakis M, Alexiou P, Grosse I and Hatzigeorgiou AG: Functional microRNA targets in protein coding sequences. Bioinformatics 28: 771-776, 2012.

42. Livak KJ and Schmittgen TD: Analysis of relative gene expression data using real-time quantitative PCR and the 2(-Delta Delta C(T)) Method. Methods 25: 402-408, 2001.

43. Alexander RP, Fang G, Rozowsky J, Snyder M and Gerstein MB: Annotating non-coding regions of the genome. Nat Rev Genet 11: 559-571, 2010.

44. Mercer TR, Dinger ME and Mattick JS: Long non-coding RNAs: Insights into functions. Nat Rev Genet 10: 155-159, 2009.

45. Mei Y, Yang JP and Qian CN: For robust big data analyses: A collection of 150 important pro-metastatic genes. Chin J Cancer 36: 16, 2017.

46. Qian CN, Mei Y and Zhang J: Cancer metastasis: Issues and challenges. Chin J Cancer 36: 38, 2017.

47. Carthew RW and Sontheimer EJ: Origins and mechanisms of miRNAs and siRNAs. Cell 136: 642-655, 2009.

48. He L and Hannon GJ: MicroRNAs: Small RNAs with a big role in gene regulation. Nat Rev Genet 5: 522-531, 2004.

49. Caiment F, Gaj S, Claessen S and Kleinjans J: High-throughput data integration of RNA-miRNA-circRNA reveals novel insights into mechanisms of benzo[a]pyrene-induced carcinogenicity. Nucleic Acids Res 43: 2525-2534, 2015.

50. Xia S, Feng J, Lei L, Hu J, Xia L, Wang J, Xiang Y, Liu L, Zhong S, Han L, et al: Comprehensive characterization of tissuespecific circular RNAs in the human and mouse genomes. Brief Bioinform 18: 984-992, 2017.

51. Salzman J, Chen RE, Olsen MN, Wang PL and Brown PO: Cell-type specific features of circular RNA expression. PLoS Genet 9: e1003777, 2013.

52. Memczak S, Jens M, Elefsinioti A, Torti F, Krueger J, Rybak A, Maier L, Mackowiak SD, Gregersen LH, Munschauer M, et al: Circular RNAs are a large class of animal RNAs with regulatory potency. Nature 495: 333-338, 2013.

53. Granados-Riveron JT and Aquino-Jarquin G: The complexity of the translation ability of circRNAs. Biochim Biophys Acta 1859: 1245-1251, 2016

54. Wang Y and Wang Z: Efficient backsplicing produces translatable circular mRNAs. RNA 21: 172-179, 2015.

55. Sannigrahi MK, Sharma R, Panda NK and Khullar M: Role of non-coding RNAs in head and neck squamous cell carcinoma: A narrative review. Oral Dis: Sep 21, 2017 (Epub ahead of print). doi: 10.1111/odi.12782.

56. Koshizuka K, Hanazawa T, Kikkawa N, Katada K, Okato A, Arai T, Idichi T, Osako Y, Okamoto Y and Seki N: Antitumor miR-150-5p and miR-150-3p inhibit cancer cell aggressiveness by targeting SPOCK1 in head and neck squamous cell carcinoma. Auris Nasus Larynx 45: 854-865, 2018.

57. Liu F, Zhao X, Qian Y, Zhang J, Zhang Y and Yin R: miR-206 inhibits head and neck squamous cell carcinoma cell progression by targeting HDAC6 via PTEN/AKT/mTOR pathway. Biomed Pharmacother 96: 229-237, 2017.

58. Miao L, Wang L, Zhu L, Du J, Zhu X, Niu Y, Wang R, Hu Z, Chen N, Shen H, et al: Association of microRNA polymorphisms with the risk of head and neck squamous cell carcinoma in a Chinese population: A case-control study. Chin J Cancer 35: 77, 2016. 
59. Chen L, Zhang S, Wu J, Cui J, Zhong L, Zeng L and Ge S: circRNA_100290 plays a role in oral cancer by functioning as a sponge of the miR-29 family. Oncogene 36: 4551-4561, 2017.

60. Rauhala HE, Jalava SE, Isotalo J, Bracken H, Lehmusvaara S, Tammela TL, Oja H and Visakorpi T: miR-193b is an epigenetically regulated putative tumor suppressor in prostate cancer. Int J Cancer 127: 1363-1372, 2010.

61. Leivonen SK, Rokka A, Ostling P, Kohonen P, Corthals GL, Kallioniemi O and Perälä M: Identification of miR-193b targets in breast cancer cells and systems biological analysis of their functional impact. Mol Cell Proteomics 10: M110.005322, 2011.

62. Mitra AK, Chiang CY, Tiwari P, Tomar S, Watters KM, Peter ME and Lengyel E: Microenvironment-induced downregulation of miR-193b drives ovarian cancer metastasis. Oncogene 34: 5923-5932, 2015.

63. Zhao GY, Ding JY, Lu CL, Lin ZW and Guo J: The overexpression of 14-3-3 $\zeta$ and Hsp27 promotes non-small cell lung cancer progression. Cancer 120: 652-663, 2014.

64. Rehman SK, Li SH, Wyszomierski SL, Wang Q, Li P, Sahin O, Xiao Y, Zhang S, Xiong Y, Yang J, et al: $14-3-3 \zeta$ orchestrates mammary tumor onset and progression via miR-221-mediated cell proliferation. Cancer Res 74: 363-373, 2014.

65. Li J, Kong F, Wu K, Song K, He J and Sun W: miR-193b directly targets STMN1 and UPA genes and suppresses tumor growth and metastasis in pancreatic cancer. Mol Med Rep 10: 2613-2620, 2014.

66. Mets E, Van der Meulen J, Van Peer G, Boice M, Mestdagh P, Van de Walle I, Lammens T, Goossens S, De Moerloose B, Benoit Y, et al: MicroRNA-193b-3p acts as a tumor suppressor by targeting the MYB oncogene in T-cell acute lymphoblastic leukemia. Leukemia 29: 798-806, 2015.

67. Zhang J, Qin J and Su Y: miR-193b-3p possesses anti-tumor activity in ovarian carcinoma cells by targeting p21-activated kinase 3. Biomed Pharmacother 96: 1275-1282, 2017.

68. Matta A, Bahadur S, Duggal R, Gupta SD and Ralhan R: Overexpression of 14-3-3zeta is an early event in oral cancer. BMC Cancer 7: 169, 2007.
69. Nishimura Y, Komatsu S, Ichikawa D, Nagata H, Hirajima S, Takeshita H, Kawaguchi T, Arita T, Konishi H, Kashimoto K, et al: Overexpression of YWHAZ relates to tumor cell proliferation and malignant outcome of gastric carcinoma. Br J Cancer 108: 1324-1331, 2013.

70. Murata T, Takayama K, Urano T, Fujimura T, Ashikari D, Obinata D, Horie-Inoue K, Takahashi S, Ouchi Y, Homma Y, et al: 14-3-3zeta, a novel androgen-responsive gene, is upregulated in prostate cancer and promotes prostate cancer cell proliferation and survival. Clin Cancer Res 18: 5617-5627, 2012.

71. Lin M, Morrison CD, Jones S, Mohamed N, Bacher J and Plass C: Copy number gain and oncogenic activity of YWHAZ/14-3-3zeta in head and neck squamous cell carcinoma. Int J Cancer 125: 603-611, 2009.

72. Yao CB, Zhou X, Chen CS and Lei QY: The regulatory mechanisms and functional roles of the Hippo signaling pathway in breast cancer. Yi Chuan 39: 617-629, 2017.

73. Patel SH, Camargo FD and Yimlamai D: Hippo signaling in the liver regulates organ size, cell fate, and carcinogenesis. Gastroenterology 152: 533-545, 2017.

74. Ji XY, Zhong G and Zhao B: Molecular mechanisms of the mammalian Hippo signaling pathway. Yi Chuan 39: 546-567, 2017.

75. Pan D: The hippo signaling pathway in development and cancer. Dev Cell 19: 491-505, 2010

76. Zhang B, Gong A, Shi H, Bie Q, Liang Z, Wu P, Mao F, Qian H and Xu W: Identification of a novel YAP-14-3-3 $\zeta$ negative feedback loop in gastric cancer. Oncotarget 8: 71894-71910, 2017.

This work is licensed under a Creative Commons Attribution-NonCommercial-NoDerivatives 4.0 International (CC BY-NC-ND 4.0) License. 